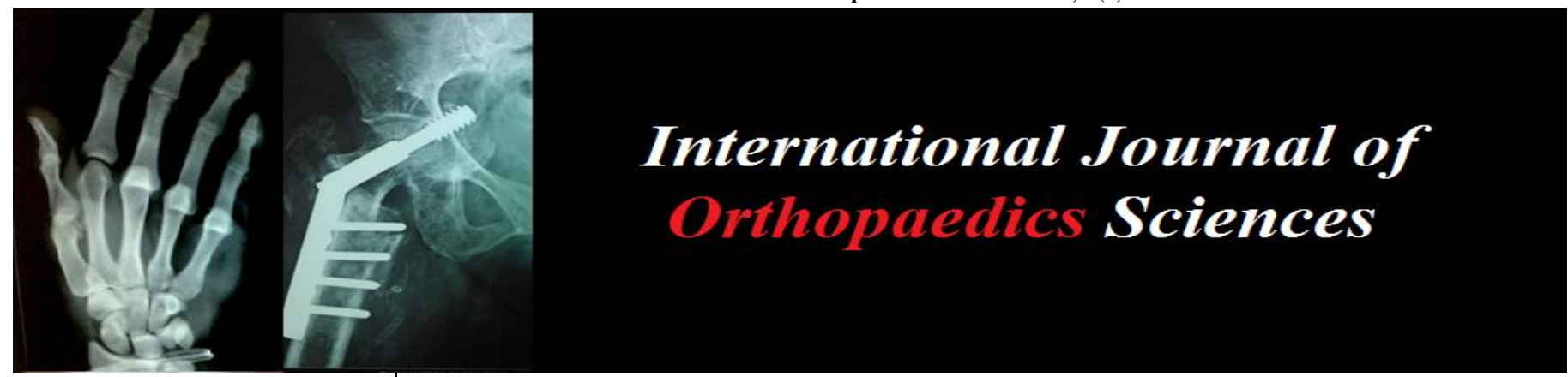

E-ISSN: 2395-1958

P-ISSN: 2706-6630

IJOS 2019; 5(3): 05-07

(C) 2019 IJOS

www.orthopaper.com

Received: 04-08-2019

Accepted: 08-09-2019

Dr. Prashant Kumar

Senior resident, Department of

Orthopaedics, VIMS,

Ramakrishna Mission Seva

Pratishthan, Kolkata, West

Bengal, India

Prof. Dipak Kumar Jha

Professor and Head of

Department of Orthopaedics,

VIMS, Ramakrishna Mission

Seva Pratishthan, Kolkata,

West Bengal, India
Corresponding Author: Dr. Prashant Kumar

Senior Resident, Department of

Orthopaedics, VIMS,

Ramakrishna Mission Seva

Pratishthan, Kolkata, West

Bengal, India

\section{Comparative study between percutaneous release and local steroid injection in trigger finger}

\section{Dr. Prashant Kumar and Prof. Dipak Kumar Jha}

DOI: https://doi.org/10.22271/ortho.2019.v5.i4a.1637

\section{Abstract}

Trigger finger is the commonest form of tendinopathy. In this study the comparison is done with two effective methods of management which is by giving steroid injection and percutaneous release.in this study two group were created of 40 patients in each group. Both the procedure was done in OPD day care basis. Patients were followed up to 6 months in gap of 1,3,6,12,24 weeks. The outcome was measured by VAS score and recurrence.

Keywords: percutaneous release, local steroid injection, trigger finger

\section{Introduction}

Trigger fingers are a common tendinopathy. The lifetime prevalence of trigger fingers among non-diabetics is approximately $2.6 \%{ }^{[1]}$. Trigger fingers typically thicken at the first annular (A1) pulley and result in incompatibility between the digital flexor tendon and its sheath ${ }^{[2]}$. The fibro-osseous canal of the A1 pulley restricts the flexor tendon excursion and causes painful triggering in the fingers. With continued deterioration, the finger may present inability to extend actively and eventually lock in flexion. Over time, a patient tends to avoid a painful trigger finger, resulting in the development of a secondary proximal interphalangeal flexion contracture $^{[3]}$. Various methods of treatment are present like, physical therapy, NSAIDS, local steroid injection, and open release. In all these methods local steroid injection is most popular method, due its effective short-term pain relief and its patient compliance. But in case of diabetes patients its sort term increases of blood sugar level, chance of infection and recurrence rate challenge its efficacity. Open release is also one of effective method, but it is cumbersome to the patients. Percutaneous release (PR), which was first introduced in 1958, has become a convenient and effective surgical procedure. It is a simple procedure can be done in OPD basis and give satisfaction to patients immediately. In this study the comparison has been done between two effective methods of dealing one of the commonest problems which is trigger finger.

\section{Methods}

\subsection{Study population, inclusion and exclusion criteria}

The study was conducted in the OPD of orthopaedics department in Vivekananda Institute of Medical Sciences, Kolkata from to, the study includes 80 patients which were divided in 2 group by randomisation. Local steroid injection was given to one group and percutaneous release was done in another. The inclusion criteria were patients exhibiting symptoms greater than Grade 2 and those who have not received any prior local injection or surgical release. The exclusion criteria were trigger finger of grade 1 and any post traumatic or post inflammatory arthritic finger. All the procedure was done by same surgeon in OPD.

\subsection{Intervention Method}

2.2.1 Method of percutaneous release: Prepare the site in a sterile fashion with povidoneiodine solution. Local infiltration of lidocaine of $1 \mathrm{ml}$ done. 18-gauge hypodermic needle and inserted it into the level of the A1 pulley (skin crease of the metacarpal joint) at a proper depth. The needle was moved longitudinally and parallel to cut the flexor tendon sheath. 
After the division of the A1 pulley, we performed a compression-confirming test (CCT)-by pressing at the A1 pulley and instructing the patients to flex and extend their affected fingers-to determine any incomplete release sites. If the flexor glided smoothly, we could determine that the A1 pulley was clinically divided completely.

2.2.2 Method of local steroid injection: Prepare the site in a sterile fashion with povidone-iodine solution. Using a 16- or 18-gauge needle attached to the 5-mL syringe, draw up a combination of $1 \mathrm{~mL}$ of lidocaine and $1 \mathrm{~mL}$ of corticosteroid (triamcinolone $40 \mathrm{mg} / \mathrm{ml}$ ). Next, change to a 25 -gauge needle. Place the needle in the midline of the finger, through the finger flexion crease at the base of the finger and angled approximately $50^{\circ}$. Advance the needle through both flexor tendons until it contacts bone. Slowly withdraw the needle, with forward pressure on the barrel of the syringe, until the resistance encountered by the needle is decreased, indicating that the needle is within the flexor sheath. This injection should not require any force, and the solution should be quite easily injected into the flexor sheath

\section{Results}

The mean age group of this study was 38.6 years. $59.4 \%$ were female whereas $39.6 \%$ were male. We observed that $35.6 \%$ of ring finger, $25.5 \%$ of thumb, $20.4 \%$ of middle finger, $14.3 \%$ of index finger and $4.2 \%$ of little finger were affected. Based on severity of disease, grading was done. $64.4 \%$ were fall in grade $2,30.4 \%$ in grade 3 and $5.2 \%$ of cases in grade 4 . This grading was further divided in 2 groups according to our study. In group of steroid injection $60 \%$ were in grade 2, $33.3 \%$ in grade 3 and $6.7 \%$ in grade 4 . In group of percutaneous release $66.6 \%$ were in grade $2,26.6 \%$ in grade 3 and $6.8 \%$ in grade 4 . The outcome was compared by VAS score in subsequent visits, immediate effectiveness of intervention and recurrence rate after 6 months. The mean
VAS score of steroid groups in the interval of 1,3,6,12,24 weeks was 2.2, 2.4, 2.5, 2.8 and 3.2 whereas the VAS score of percutaneous release group was $4.4,3.8,3.6,3.4$ and 3.3 respectively. The immediate effectiveness of intervention in term of relief of triggering in steroid group was $38 \%$ in grade 2 and $0 \%$ in grade 3 and grade 4, whereas in group of percutaneous release group $100 \%$ relief in grade 2, 3 and 4.The recurrence rate was evaluated after 6 months interval, which showed $50 \%$ of recurrence in grade 2 of steroid group whereas $10 \%$ in grade $2,12 \%$ in grade 3 and $50 \%$ recurrence in group of percutaneous release. There were no neurapraxia, tendon bowstring, infection and vascular injury in this study. Only 2 patients of steroid group have faced hypopigmentation at injection site.

\section{Discussion}

Steroid injection has been a popular and easy method to deal with trigger finger, but the problem of triggering always compromised its effectiveness. In our study ring finger was most common digits to be affected but in the study of Zyluk et $a l .{ }^{[4]}$, triggering of thumb was the most common one. After intervention VAS score of percutaneous release group in our study was $4.4 \pm 1.5$ which can be compared with study of Niraj et al. ${ }^{[5]}$. of $4.7 \pm 2.2$. VAS score in steroid group decreased from $8.4 \pm 1.5$ to $2.2 \pm 1$ which is compared with $7.3 \pm 0.9$ to $1.7 \pm 0.4$ in study of Yutaka Mifune et al. ${ }^{[6]}$ after 1week interval. The immediate satisfaction level to the patients was loud and clear in the case of percutaneous release with $100 \%$ results, which can be compared which Chao and colleague $^{[7]}$ and Zyluk et al. of $96 \%$ and $100 \%$ respectively. The recurrence rate of triggering is $50 \%$ with steroid injection in grade 2 of trigger finger whereas its only $10 \%$ with percutaneous release. Percutaneous release is a minimally invasive surgery which can be done in OPD basis and having similar success rate as and low complication compared with open release.

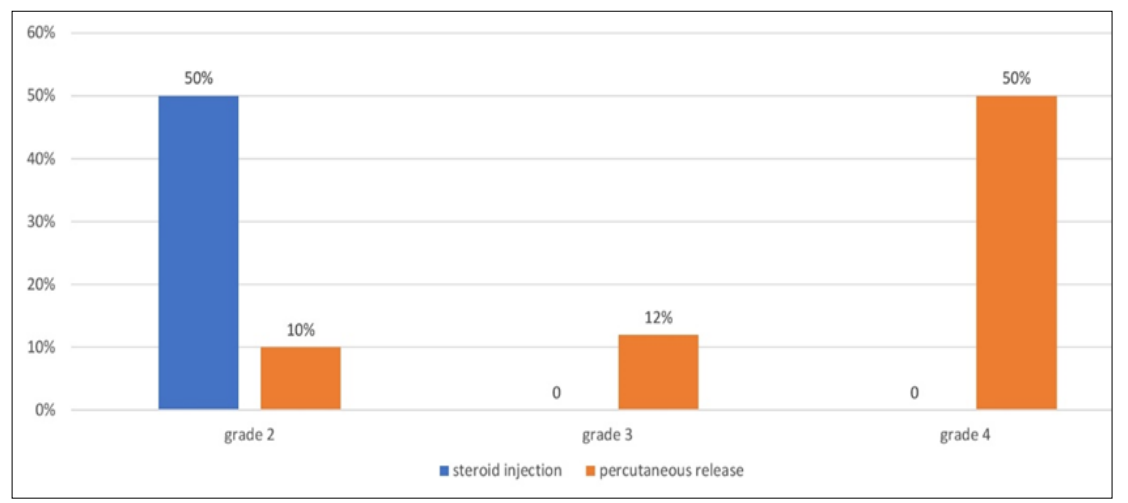

Fig 1: Recurrence rate

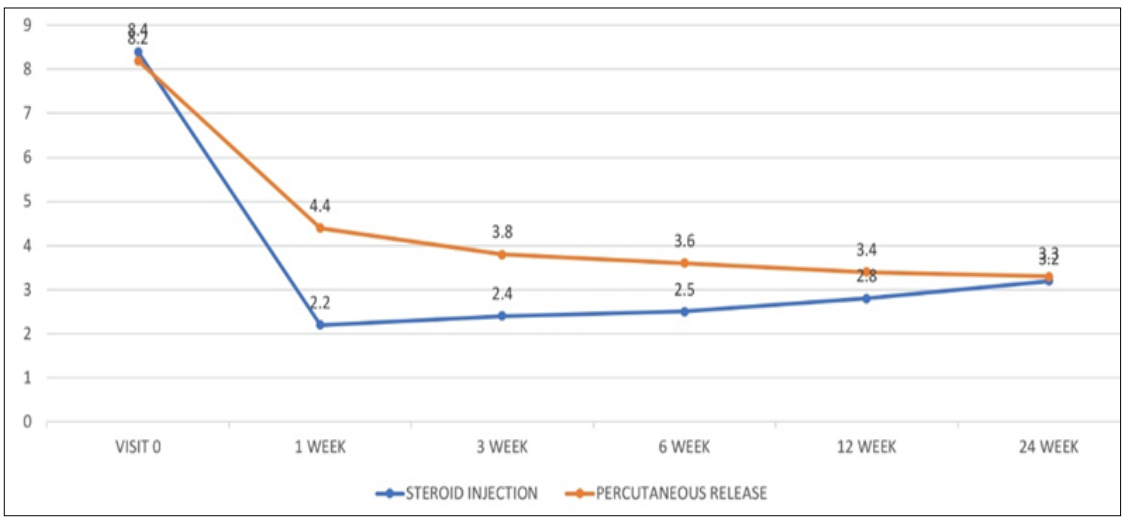

Fig 2: VAS score 


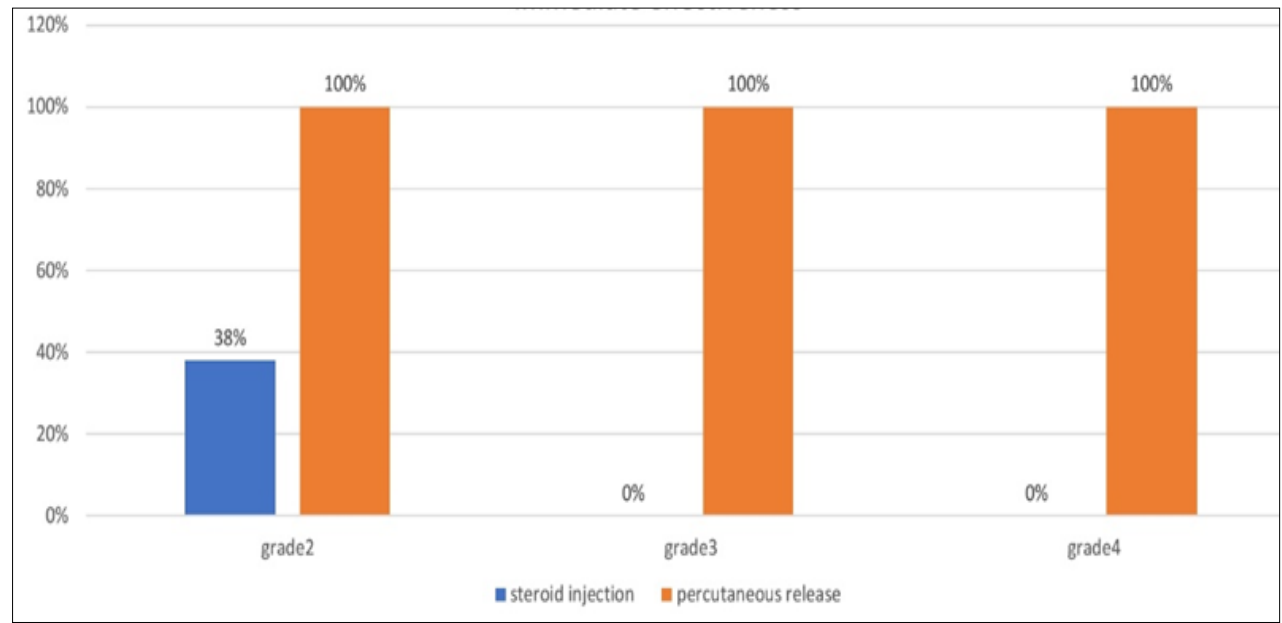

Fig 3: Immediate effectiveness

\section{Conclusion}

Steroids injection is an effective treatment in grade 0 and 1 where main problem is pain, whereas percutaneous release is a well effective method to treat grade 2 and 3.Steroids can reduce VAS score significantly as compare with percutaneous release immediately but in long term VAS score is almost equal. Percutaneous release is a well effective method to treat grade 2 and 3.Open release will be a preferred one in grade 4 due to high recurrence rate in percutaneous release. We will recommend using steroid injection in grade 0 and 1, whereas to combine steroid injection with percutaneous release in grade 2 and 3 and open release for grade 4 of trigger finger.

\section{Conflicts of interest: No conflicts of interest}

\section{References}

1. Wojahn RD, Foeger NC, Gelberman RH, Calfee RP. Long-term outcomes following a single corticosteroid injection for trigger finger J Bone Joint Surg Am. 2014; 96:1849-1854.

2. Sato ES, Gomes Dos Santos JB, Belloti JC, Albertoni WM, Faloppa F. Treatment of trigger finger: randomized clinical trial comparing the methods of corticosteroid injection, percutaneous release and open surgery Rheumatology (Oxford). 2012; 51:93-99.

3. Makkouk AH, Oetgen ME, Swigart CR, Dodds SD. Trigger finger: etiology, evaluation, and treatment Curr Rev Musculoskelet Med. 2008; 1:92-96.

4. Zyluk A, Jagielski G. Percutaneous A1 pulley release vs steroid injection for trigger digit: the results of a prospective, randomized trial. J Hand Surg Eur. 2011; 36:53-6.

5. Niraj Ranjeet, Krishna Sapkota, Pabin Thapa, Trigger Finger. A Prospective Randomised Control Trial Comparing Percutaneous Release versus Open Release Journal of Clinical and Diagnostic Research. 2018; 12(7):RC05-RC08.

6. Yutaka Mifune. Atsuyuki Inui. Ryosuke Ssakata. Highresolution ultrasound in the diagnosis of trigger finger and evaluation of response to steroid. Skeletal Radiol DOI: $10.1007 / \mathrm{s} 00256-016-2485-5$

7. Chao M, Wu S, Yan T. The effect of miniscalpel-needle versus steroid injection for trigger thumb release. J Hand Surg Eur Vol. 2009; 34(4):522-5. 